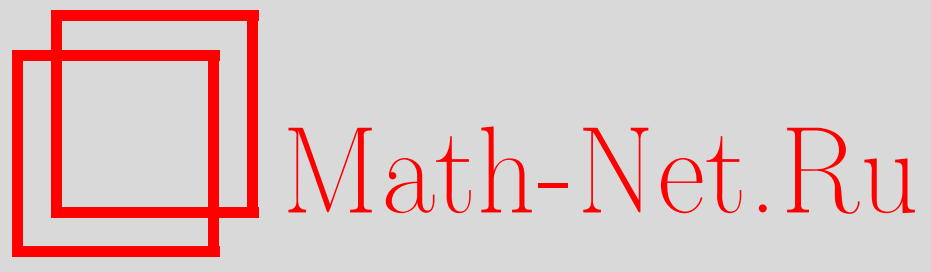

А. А. Гайфуллин, Явное построение многообразий, реализующих заданные классы гомологий, УМН, 2007, том 62, выпуск 6, 167-168

DOI: https://doi.org/10.4213/rm8537

Использование Общероссийского математического портала Math-Net.Ru подразумевает, что вы прочитали и согласны с пользовательским соглашением http://www . mathnet.ru/rus/agreement

Параметры загрузки:

IP : 3.80 .253 .173

26 апреля 2023 г., 15:59:41

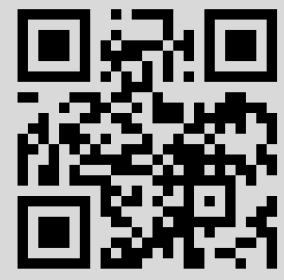




\section{Явное построение многообразий, реализующих заданные классы гомологий}

\section{А. А. Гайфуллин}

Хорошо известна задача Н. Стинрода о реализации классов гомологий образами фундаментальных классов многообразий. В настоящей заметке мы даем явную комбинаторную конструкцию, которая по циклу строит кусочно линейное многообразие, реализующее с некоторой кратностью целочисленный класс гомологий этого цикла. Конструкция основана на процедуре локального разрешения особенностей цикла. Подход к проблеме Н. Стинрода, основанный на разрешении особенностей псевдомногообразий, был впервые предложен Д. Сулливаном [1]. Построенные им препятствия к разрешению особенностей псевдомногообразия являются элементами конечного порядка, поэтому из результатов [1] легко следует, что разрешение особенностей "с некоторой кратностью" возможно всегда. Наша задача заключается в том, чтобы дать явную конструкцию такого разрешения особенностей.

Нам будет удобно работать с псевдомногообразиями, разбитыми на простые клет$\kappa u$. Простая клетка - это замкнутый диск, граница которого наделена разбиением на грани, двойственным кусочно линейной триангуляции сферы. Строгие определения простой клетки и разбиения на простые клетки можно найти в [2]. Примерами разбиений на простые клетки служат симплициальные и кубические разбиения, а также клеточное разбиение, двойственное кусочно линейной триангуляции многообразия. Все многообразия и псевдомногообразия предполагаются замкнутыми.

Пусть $Z$ - ориентированное $n$-мерное псевдомногообразие, разбитое на простые клетки. Задача разрешения особенностей псевдомногообразия $Z$ : построить ориентированное кусочно линейное многообразие $M$ и отображение $g: M \rightarrow Z$ такие, что над дополнением к $(n-2)$-мерному остову разбиения $Z$ отображение $g$ является конечнолистным накрытием.

Центральная конструкция. Пусть каждой $(n-1)$-мерной клетке разбиения $Z$ сопоставлена метка из некоторого конечного множества. Множество меток $(n-1)$-мерных клеток, содержащих клетку $F$, мы будем называть меткой клетки $F$ и обозначать через $c(F)$. Разметку мы будем называть хорошей, если $|c(F)|=n-\operatorname{dim} F$ для любой клетки $F$, причем для каждой $n$-мерной клетки $G \supset F$ метки всех $(n-1)$-мерных клеток $H$ таких, что $F \subset H \subset G$, попарно различны.

ЛЕмма. Для каждого псевдомногообразия $Z$, разбитого на простые клетки, имеются псевдомногообразие $\bar{Z}$ с разбиением на простые клетки, допускающим хорошую разметку, и отображение $\bar{Z} \rightarrow Z$, являющееся накрытием на дополнении $\kappa$ остову коразмерности 2 и отображающее каждую клетку разбиения $\bar{Z}$ изоморбно на некоторую клетку разбиения $Z$.

Таким образом, в дальнейшем мы можем считать, что на разбиении $Z$ задана хорошая разметка. Пусть $F-$ клетка разбиения $Z, \operatorname{dim} F=k<n$. Обозначим через $L_{F}$ множество $n$-мерных клеток, содержащих $F$. Из наличия хорошей разметки следует, что клетки $G \in L_{F}$ могут быть раскрашены в два цвета правильным образом, т. е. так, что никакие две клетки одного цвета не имеют общей гиперграни. При этом количество клеток первого цвета равно количеству клеток второго цвета. Обозначим через $P_{F}$ множество инволюций на множестве $L_{F}$, изменяющих цвета всех клеток на противоположные. Обозначим через $P$ декартово произведение множеств $P_{F}$ по всем клеткам $F$ разбиения $Z$.

Обозначим через $U$ множество наборов $\left(F_{0}, F_{1}, \ldots, F_{n}\right)$, где $F_{0} \supset F_{1} \supset \cdots \supset F_{n}-$ клетки разбиения $Z$ и $\operatorname{dim} F_{i}=n-i$. Положим $V=U \times P \times \mathbb{Z}_{2}^{n}$.

Работа выполнена при поддержке гранта РФФИ № 05-01-01032 и гранта НШ-4182.2006.1. 
Определим инволюции $\Phi_{j}^{\varepsilon}: V \rightarrow V, \varepsilon=0,1, j=1, \ldots, n$, по формулам

$$
\begin{gathered}
\Phi_{j}^{0}\left(F_{0}, F_{1}, \ldots, F_{n},\left(\Lambda_{F}\right), h\right)=\left(F_{0}, F_{1}, \ldots, F_{j-1}, F_{j}^{*}, F_{j+1}, \ldots, F_{n},\left(\Lambda_{F}\right), h\right), \\
\Phi_{j}^{1}\left(F_{0}, F_{1}, \ldots, F_{n},\left(\Lambda_{F}\right), h\right)=\left(\widetilde{F}_{0}, \widetilde{F}_{1}, \ldots, \widetilde{F}_{n},\left(\widetilde{\Lambda}_{F}\right), h+e_{j}\right),
\end{gathered}
$$

где $\Lambda_{F} \in P_{F}, h \in \mathbb{Z}_{2}^{n},\left(e_{1}, \ldots, e_{n}\right)$ - базис группы $\mathbb{Z}_{2}^{n}$ и

1) $F_{j}^{*}$ - единственная отличная от $F_{j}$ клетка такая, что $F_{j-1} \subset F_{j}^{*} \subset F_{j+1}$;

2) $\widetilde{F}_{i}=F_{i}$ при $i \geqslant j ; \widetilde{F}_{0}=\Lambda_{F_{j}}\left(F_{0}\right)$; если $0<i<j$, то $\widetilde{F}_{i}-$ единственная клетка такая, что $\widetilde{F}_{j} \subset \widetilde{F}_{i} \subset \widetilde{F}_{0}$ и $c\left(\widetilde{F}_{i}\right)=c\left(F_{i}\right)$;

3) если $c(F) \not \supset c\left(F_{j}\right)$, то $\widetilde{\Lambda}_{F}=\Lambda_{F}$;

4) если $c(F) \supset c\left(F_{j}\right)$ и $G \in L_{F}$, то $\widetilde{\Lambda}_{F}(G)=\left(\Lambda_{H_{2}} \circ \Lambda_{F} \circ \Lambda_{H_{1}}\right)(G)$, где $H_{1}$ - единственная клетка такая, что $F \subset H_{1} \subset G$ и $c\left(H_{1}\right)=c\left(F_{j}\right)$, и $H_{2}$ - единственная клетка такая, что $F \subset H_{2} \subset \Lambda_{F}\left(\Lambda_{H_{1}}(G)\right)$ и $c\left(H_{2}\right)=c\left(F_{j}\right)$.

Положим $M=[0,1]^{n} \times V / \sim$, где $\sim$ - отношение эквивалентности, порожденное отождествлениями $\left(t_{1}, \ldots, t_{n}, \Phi_{j}^{\varepsilon}(v)\right) \sim\left(t_{1}, \ldots, t_{n}, v\right)$, если $t_{j}=\varepsilon, \varepsilon=0,1, j=1, \ldots, n$. Непосредственно проверяется, что $\Phi_{j}^{0} \Phi_{k}^{1}=\Phi_{k}^{1} \Phi_{j}^{0}$ при $j \neq k$ и $\Phi_{j}^{1} \Phi_{k}^{1}=\Phi_{k}^{1} \Phi_{j}^{1}$. Из этого следует, что $M$ - ориентированное кусочно линейное многообразие. Искомое отображение $g: M \rightarrow Z$ определяется по формуле

$$
g\left(t_{1}, \ldots, t_{n}, F_{0}, \ldots, F_{n},\left(\Lambda_{F}\right), h\right)=\left(\prod_{i=1}^{n}\left(1-t_{i}\right)\right) b\left(F_{0}\right)+\sum_{j=1}^{n}\left(t_{j} \prod_{i=j+1}^{n}\left(1-t_{i}\right)\right) b\left(F_{j}\right),
$$

где $b(F)$ - барицентр клетки $F$.

Таким образом, мы получаем следующий результат.

Теорема 1. Центральная конструкиия дает отображение $g: M \rightarrow Z$, решающее задачу разрешения особенностей псевдомногообразия $Z$. Пусть $X$ - топологическое пространство и $f: Z \rightarrow X$ - сингулярный иикл. Тогда сквозное отображение $f \circ g$ : $M \rightarrow X$ реализует класс гомологий, кратный классу гомологий изила $f$.

В качестве приложения теоремы 1 дадим решение следующей задачи: по заданному набору ориентированных симплициальных сфер $Y_{1}, \ldots, Y_{k}$ построить ориентированное симплициальное многообразие, набор линков вершин которого совпадает с точностью до изоморфизма с набором $Y_{1}, \ldots, Y_{k}$. Для существования такого многообразия необходимо, чтобы вершины триангуляций $Y_{1}, \ldots, Y_{k}$ могли быть разбиты на пары такие, что линки вершин в каждой паре изоморфны с обращением ориентации (условие сбалансированности).

Теорема 2. Пусть $Y_{1}, \ldots, Y_{k}$ - сбалансированный набор ориентированных симплициальных сфер. Имеется явная конструкиия, дающая симплициальное многообразие, набор линков вериин которого с точностью до изоморфизма имеет вид

$$
\underbrace{Y_{1}, \ldots, Y_{1}}_{r}, \ldots, \underbrace{Y_{k}, \ldots, Y_{k}}_{r}, K_{1}, \ldots, K_{l},-K_{1}, \ldots,-K_{l},
$$

где $-K_{i}$ есть симпличиальная сфера $K_{i}$ с обращенной ориентацией.

Автор благодарен В. М. Бухштаберу за постановки задач и внимание к работе.

\section{Список литературы}

[1] D. Sullivan, Lecture Notes in Math., 209, 1971, 196-206. [2] А. А. Гайфуллин, Изв. РАН. Сер. матем., 68:5 (2004), 13-66.

\section{A. А. Гайфуллин (A. A. Gaifullin)}

Московский государственный университет им. М. В. Ломоносова

E-mail: gaifull@mccme.ru
Представлено В. М. Бухштабером Принято редколлегией 08.10 .2007 